\title{
HUMAN DENTAL PULP STEM CELLS - ISOLATION AND LONG TERM CULTIVATION
}

\author{
Jakub Suchánek1, Tomáš Soukup ${ }^{2}$, Romana Ivančaková1, Jana Karbanová2, Věra Hubková ${ }^{1}$, Robert Pytlík ${ }^{3}$, \\ Lenka Kučerová ${ }^{4}$
}

Charles University in Prague, Faculty of Medicine in Hradec Králové and University Hospital Hradec Králové, Czech Republic, Department of Dentistry ${ }^{1}$, Department of Histology and Embryology ${ }^{2}$, Department of Clinical Genetics ${ }^{4}$; Charles University in Prague, $1^{\text {st }}$ Medical Faculty in Prague, Teaching Hospital, Czech Republic: $1^{\text {st }}$ Department of Medicine ${ }^{3}$

Summary: Human adult mesenchymal stem cells (MSCs) are rare elements living in various organs (e.g. bone marrow, skeletal muscle), with capability to differentiate in various cell types (e.g. chondrocytes, adipocytes and osteoblasts). In the year 2000, Gronthos and co-workers isolated stem cells from the human dental pulp (DPSCs). Later on, stem cells from exfoliated tooth were also obtained. The aims of our study were to establish protocol of DPSCs isolation and to cultivate DPSCs either from adult or exfoliated tooth, and to compare these cells with mesenchymal progenitor cell (MPCs) cultures. MPCs were isolated from the human bone marrow of proximal femur. DPSCs were isolated from deciduous and permanent teeth. Both cell types were cultivated under the same conditions in the media with $2 \%$ of FCS supplemented with PDGF and EGF growth factors. We have cultivated undifferentiated DPSCs for long time, over 60 population doublings in cultivation media designed for bone marrow MPCs. After reaching Hayflick's limit, they still have normal karyotype. Initial doubling time of our cultures was from 12 to 50 hours for first 40 population doublings, after reaching 50 population doublings, doubling time had increased to 60-90 hours. Regression analysis of uncumulated population doublings proved tight dependence of population doublings on passage number and slow decrease of proliferation potential. In comparison with bone marrow MPCs, DPSCs share similar biological characteristics and stem cell properties. The results of our experiments proved that the DPSCs and MPCs are highly proliferative, clonogenic cells that can be expanded beyond Hayflick's limit and remain cytogenetically stable. Moreover we have probably isolated two different populations of DPSCs. These DPSCs lines differed one from another in morphology. Because of their high proliferative and differentiation potential, DPSCs can become more attractive, easily accessible source of adult stem cells for therapeutic purposes.

Key words: Dental pulp; Stem cells; Isolation; Cultivation; Doubling time; Hayflick's limit

\section{Introduction}

Stem cells (SCs) are special type of cells, which can be found almost in each type of tissue and through entire life span in multicellular organism. Their main functions are to provide tissue development, homeostasis and in the case of tissue damage its reparation. In order to reach these functions, stem cells have two unique properties. The first one is their capacity of self renew beyond Hayflick's limit (it means, that the cell line is able to proliferate over approx. 50 population doublings) (4). The second ability is to differentiate into mature cell types.

Mesenchymal stem cells (MSCs) are rare elements living in various mesenchymal tissues, for example in the bone marrow stroma ( 2 to 5 cells per million of nucleated cells) (8), liver or skeletal muscles (12). Later on, more pri- mitive mesenchymal SCs were discovered. Those immunomagnetically separated cells were named mesodermal progenitor cells (MPCs) (10) or multipotent adult progenitor cells (MAPCs) (6).

In a year 2000, Gronthos and co-workers isolated stem cells from the human dental pulp (DPSCs) (3). The pulp tissue was extracted from impacted third molars in their experiment. In the year 2003, Miura at al. (7) have isolated stem cells from human exfoliated deciduous teeth (SHED).

The origin of DPSCs has not been solved yet (9). Regarding to complicated process of odontonogenesis, germs of the teeth are rising from two embryonic layers (the ectomesenchyme of neural crest and ectoderm of dental lamina) (1). Therefore, we suppose that there are two different DPSCs lines within the dental pulp. First population probably derives from neural crest mesenchyme. Second me- 
senchymal population might be derivative of the ectodermal dental lamina. However, exact localization of DPSCs inside the dental pulp was not covered up to this day.

Dental pulp is well defined compartment of soft tissue, which keeps primitive structure similar to gelatinous tissue of umbilical cord. Because of specific DPSCs niche, we propose that these cells will share some characteristics with embryonic stem cells.

According to our knowledge, no study described biological behavior and differentiation potential of DPSCs cultivated in medium optimized for human MPCs was published till now. Both, MPCs and DPSCs, represent perspective therapeutic tool of wide clinical use (cell therapy) and laboratory modelling.

\section{Methods}

Series of dental pulp and bone marrow donors. Tooth donors were divided into 2 major groups: 1) deciduous and 2) permanent teeth, which were divided into following subgroups. 1a) spontaneously exfoliated teeth and $1 \mathrm{~b}$ ) extracted deciduous teeth, in which resorption of the root did not exceed one half of the original length, 2a) impacted third molars, 2b) erupted third molars and $2 \mathrm{c}$ ) erupted premolars (5). Teeth in subgroups $1 \mathrm{~b}, 2 \mathrm{a}, 2 \mathrm{~b}$ and $2 \mathrm{c}$ were extracted often due to the orthodontic reasons, or when they caused serious health problems to patients.

Bone marrow was collected from trochanteric area of patients undergoing hip replacement.

Tab. 1: Tooth donors.

\begin{tabular}{|c|c|c|c|}
\hline $\begin{array}{l}\text { Patient } \\
\text { No. }\end{array}$ & Sex & Age & $\begin{array}{c}\text { Extracted } \\
\text { teeth }\end{array}$ \\
\hline Z6/06 & $\mathrm{F}$ & 17 & M3 \\
\hline Z7/06 & $\mathrm{F}$ & 21 & M3 \\
\hline Z8/06 & $\mathrm{F}$ & 21 & M3 \\
\hline Z12/06 & $\mathrm{F}$ & 18 & M3 \\
\hline Z13/06 & $\mathrm{F}$ & 18 & M3 \\
\hline Z14/06 & $\mathrm{F}$ & 16 & $\mathrm{P} 1$ \\
\hline Z15/06 & $\mathrm{F}$ & 23 & M3 \\
\hline Z16/06 & $\mathrm{F}$ & 19 & M3 \\
\hline Z17/06 & $\mathrm{F}$ & 12 & P1 \\
\hline Z18/06 & $\mathrm{F}$ & 19 & $\mathrm{P} 1$ \\
\hline Z19/06 & $\mathrm{F}$ & 19 & M3 \\
\hline Z20/06 & $\mathrm{F}$ & 12 & M3 \\
\hline Z21/06 & $\mathrm{F}$ & 12 & $\mathrm{P} 1$ \\
\hline Z23/06 & $\mathrm{M}$ & 15 & M3 \\
\hline Z1/07 & $\mathrm{F}$ & 21 & M3 \\
\hline Z3/07 & $\mathrm{F}$ & 22 & M3 \\
\hline Z4/07 & $\mathrm{M}$ & 16 & M3 \\
\hline Z5/07 & $\mathrm{F}$ & 20 & M3 \\
\hline Z6/07 & $\mathrm{F}$ & 20 & M3 \\
\hline Z7/07 & $\mathrm{F}$ & 17 & M3 \\
\hline Z_exfoll. & $\mathrm{F}$ & 8 & $\mathrm{i} 2$ \\
\hline Z_exfol2. & $\mathrm{M}$ & 8 & $\mathrm{i} 2$ \\
\hline
\end{tabular}

All patients or their legitimate representatives were supposed to subscribe informed consent according to guidelines of the Ethical committee of the Medical Faculty in Hradec Králové and Faculty Hospital in Hradec Králové.

Teeth were obtained from 22 healthy patients of average age 17 (8-23) (Tab. 1).

Bone marrow was aspirated from 10 patients, mostly postmenopausal women of average age 71 and one 70 years old man.

DPSCs and MPCs isolation. Third molars were obtained from 16 consecutive patients (healthy donors) undergoing third molar extraction (Fig. 1). Likewise, four premolars and two exfoliated tooth were collected. Dental pulp was isolated under the sterile conditions using different procedures for DP extraction.

If the roots had already finished the development, we splitted the crown using skive (Fig. 2) without cooling, so there was a high risk of mechanical and heat damage of the dental pulp (DP). To avoid thermic damage, diamond burr for cooling turbine was used (Fig. 3). Following tooth splitting, DP was isolated using excavator (Henry Schein Inc., UK). The tooth and the pulp were then transported in Hank's balanced salted solution (HBSS) (Gibco, Scotland) to our laboratory.

Another DP harvesting procedure was completely done in tissue cultures laboratory. Intact tooth with the pulp was transported in HBSS into laboratory. There we used Luer's forceps to break the roots in order to extract the pulp through the root canals. If the roots did not finished their development and apical foramen was widely opened, we used sharp needle to release DP from the pulp chamber. If the roots were not wide enough, we used extirpation needle.

Both, the dental pulp and tooth, were enzymatically treated with collagenase (Sevapharma, CR) and dispase (Gibco, Scotland) for 70 minutes. Cell pellet of two fractions was obtained by centrifugation: A) Cell fraction from subodontoblastic compartment (SOc) and B) Cell fraction from perivascular compartment (PVc) (Fig. 4).

Aspirated bone marrow was diluted in cooled $\left(4{ }^{\circ} \mathrm{C}\right)$ HBSS with Heparine (Léčiva, CR) and transported. Bone marrow mononuclear cells were obtained by optimized Ficoll-Paque density gradient centrifugation (11).

Culture conditions. Both, DPSCs and MPCs cell suspensions were cultivated under the same culture conditions, using previously described (11) medium for human mesenchymal progenitor cells (MPCs) composed of alphaMEM (Gibco, Scotland), 2 \% FCS (PAA, USA), EGF (PeproTech, USA), PDGF (PeproTech, USA) and dexamethasone (Sigma, USA) and in some cases supplemented with ITS supplement (Sigma, USA). DPSCs and MPCs were cultivated for 3-5 days in primary culture inside culture flasks with Cell ${ }^{+}$surface (Sarstedt, USA), then treated with trypsin-EDTA (Gibco, Scotland) and splitted into culture flasks with standard tissue cultures treated surfaces (TPP or NUNC, Denmark). Each following passaging was done after reaching $70 \%$ of confluence. 
Cell analysis. Cell viability and number of population doublings were examined using Vi-cell analyser and Z2 Counter (both from Beckman Coulter, USA). DNA analysis was done using propidium iodide staining and flow cytometry (Cell Lab Quanta, Beckman Coulter, USA). For karyotyping cells (subcultured at a 1:3 dilution, both early passages and after reaching Hayflick's limit) were after 24 hours cultivation subjected to a 4-hour Demecolcemid (Sigma, USA) incubation followed by trypsin-EDTA detachment and lysis with hypotonic $\mathrm{KCl}$ and fixation in acid/alcohol. Metaphases were analyzed after GTG banding using software Ikaros v 5.0 (MetaSystems, USA).

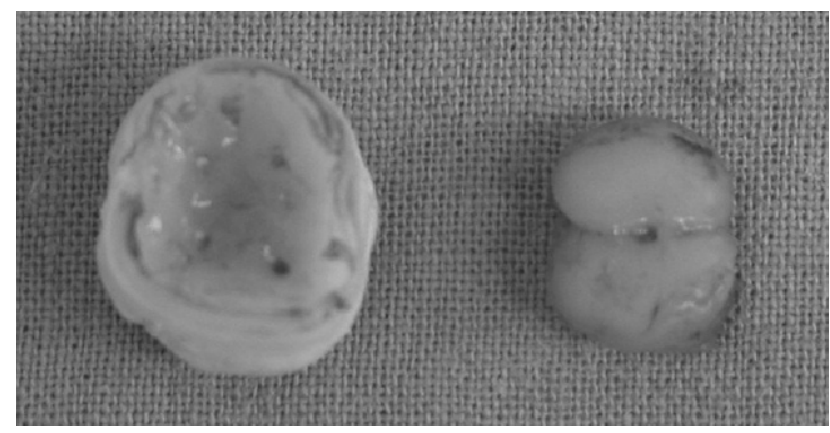

Fig. 1: Third molar after germectomy - crown and extracted dental pulp.

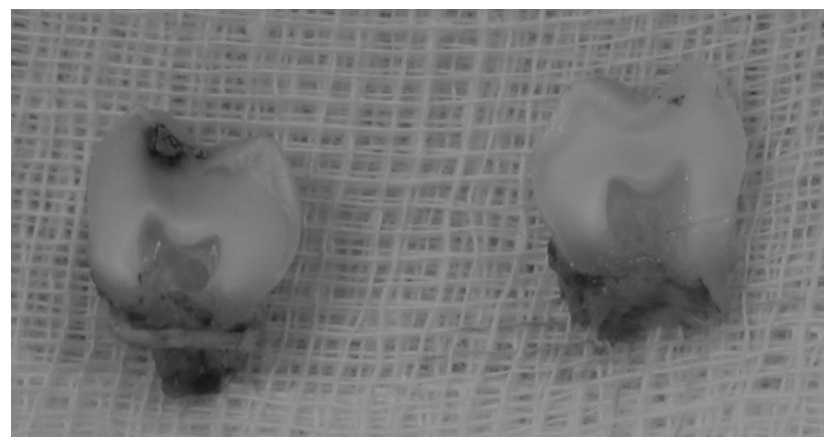

Fig. 2: Exfoliated deciduous molar - dental crown splitted using skive.

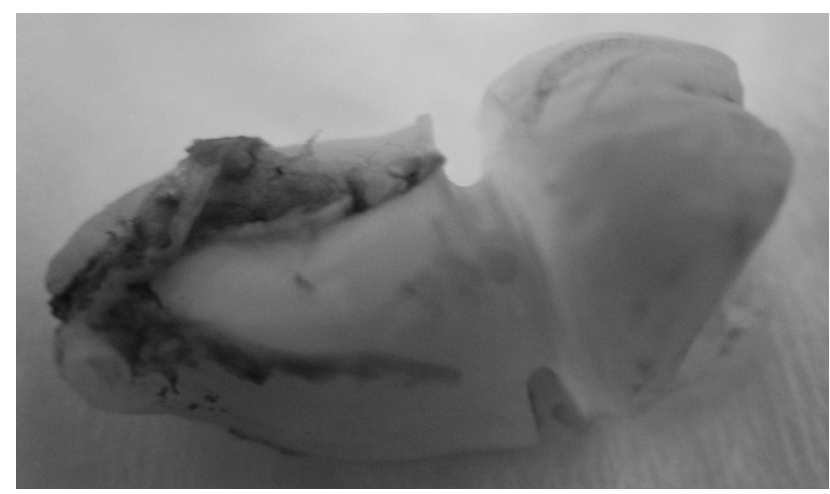

Fig. 3: Third molar with fully developed roots which was splitted using diamond burr.

\section{Results}

We were able to isolate selected DPSCs from both compartments of extracted third molars and mixed DPSCs cultures from premolars and deciduous teeth using Luer's forceps or extirpation needle. On the other hand, we were not able to isolate DPSCs from the teeth which were splitted using skive or diamond grindstone. For that reason and also because of high risk of sample contamination, we left these grinding methods.

We obtained in average $46 \pm 6$ (10-108) DPSCs using enzymatic dissociation of the dental pulp. Primary cultures

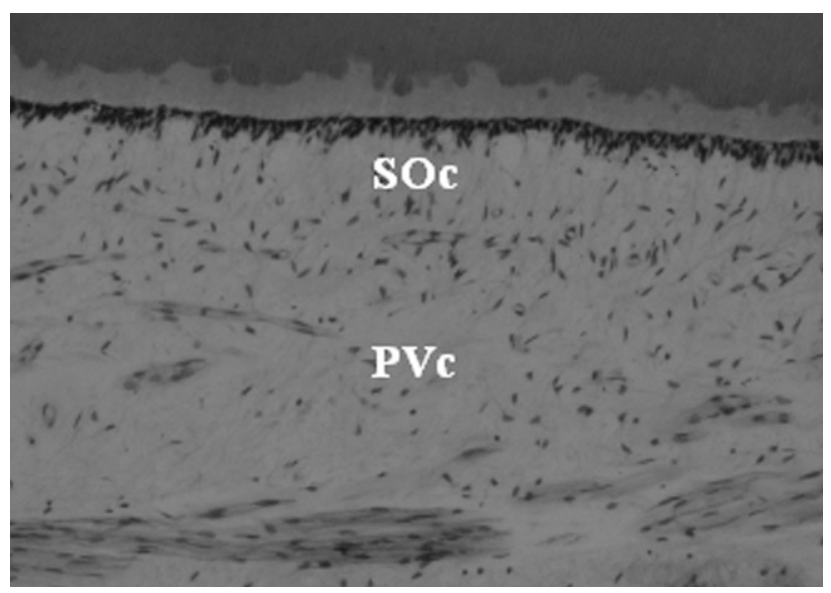

Fig. 4: Histological structure of the dental pulp with defined compartments (SOc - subodontoblastic compartment, PVc - perivascular compartment). Haematoxylin Eosin staining, direct magnification 100x.

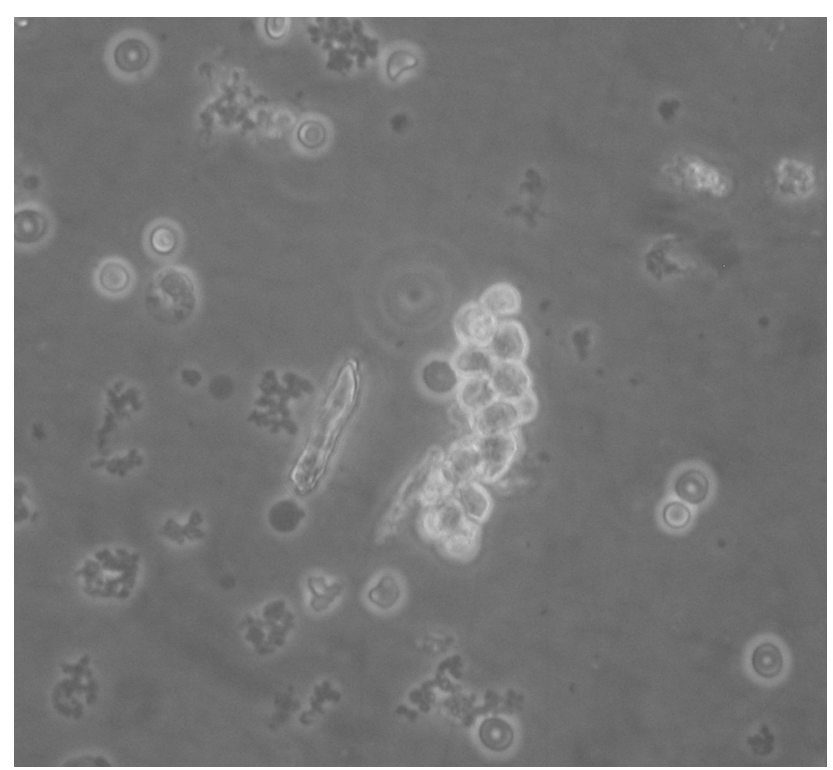

Fig. 5: Inoculated DPSCs (day 1) with remnants of the dental pulp. Phase contrast microscopy, direct magnification $200 x$. 


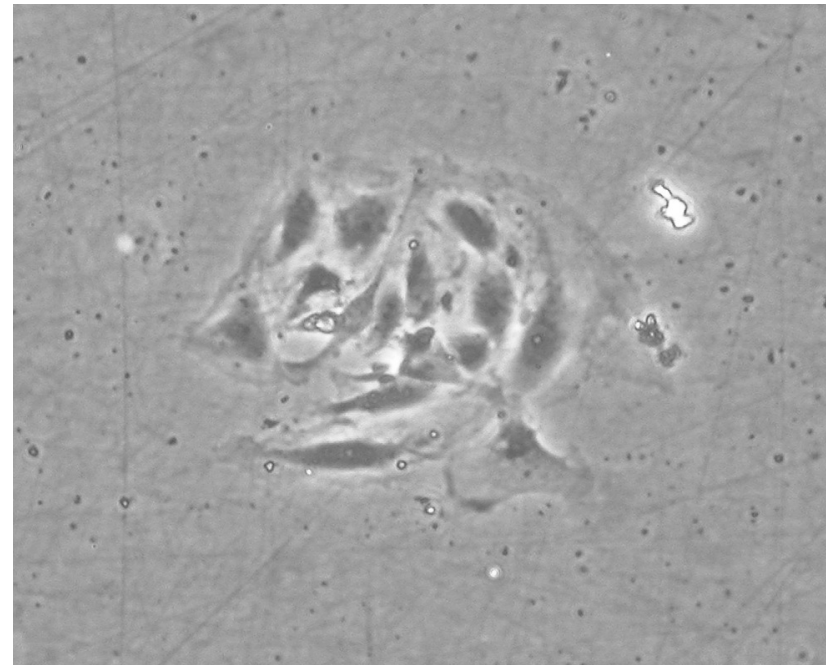

Fig. 6: Small colony of DPSCs 24 hours following inoculation. DPSCs are 12 to $18 \mu \mathrm{m}$ in diameter. Phase contrast microscopy, direct magnification 200x.

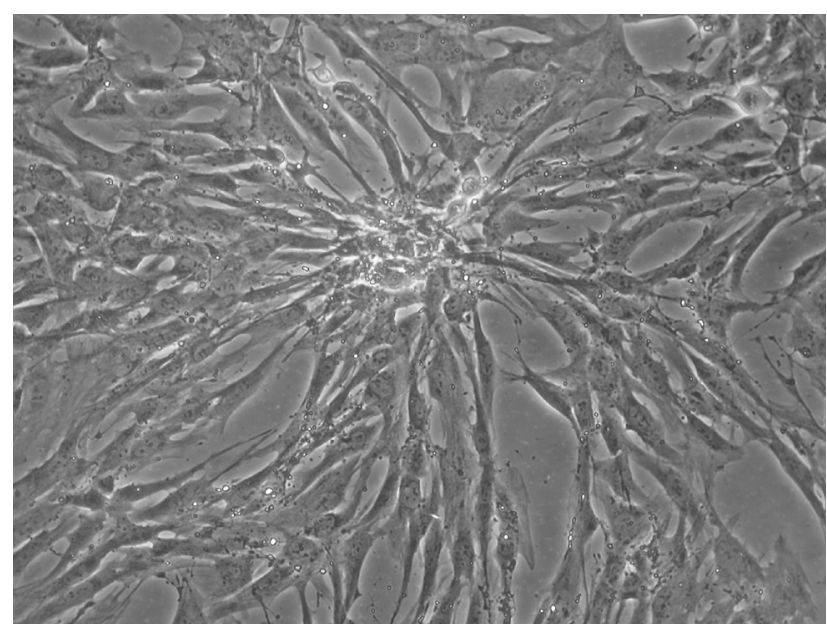

Fig. 7: DPSCs primary culture 5 days following inoculation. DPSCs are 12 to $18 \mu \mathrm{m}$ in diameter. Phase contrast microscopy, direct magnification 200x.

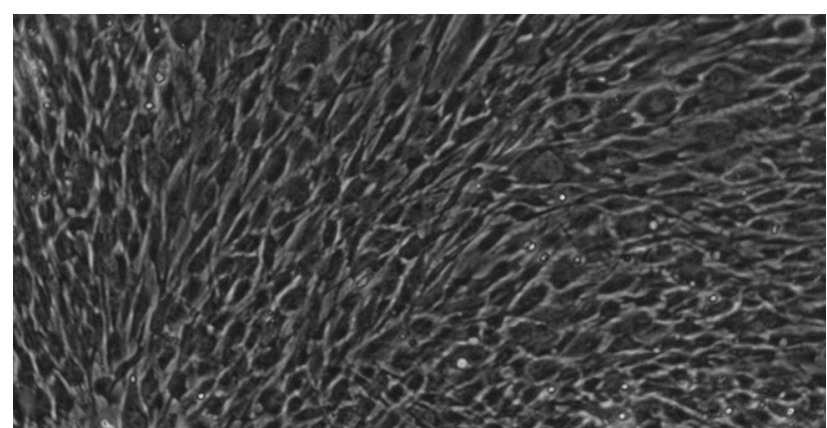

Fig. 8: DPSCs isolated from SOc (passage No. 30). DPSCs are more rounded then DPSCs isolated from PVc. Phase contrast microscopy, direct magnification 200x.

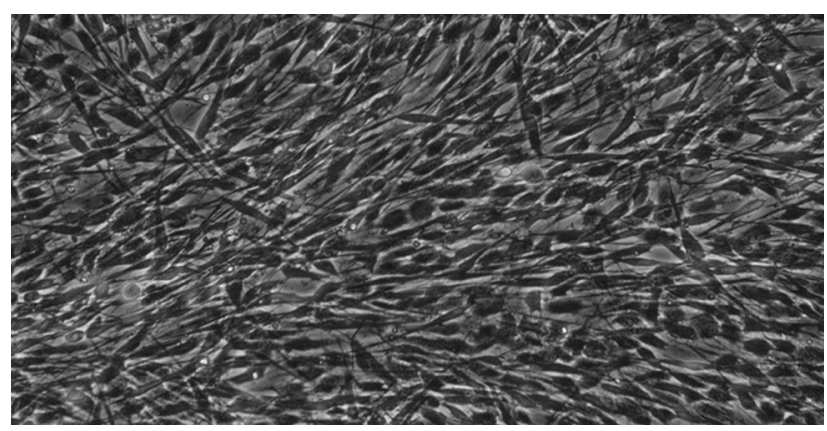

Fig. 9: Spindle shaped DPSCs isolated from PVc (passage No. 30). Phase contrast microscopy, direct magnification 200x.

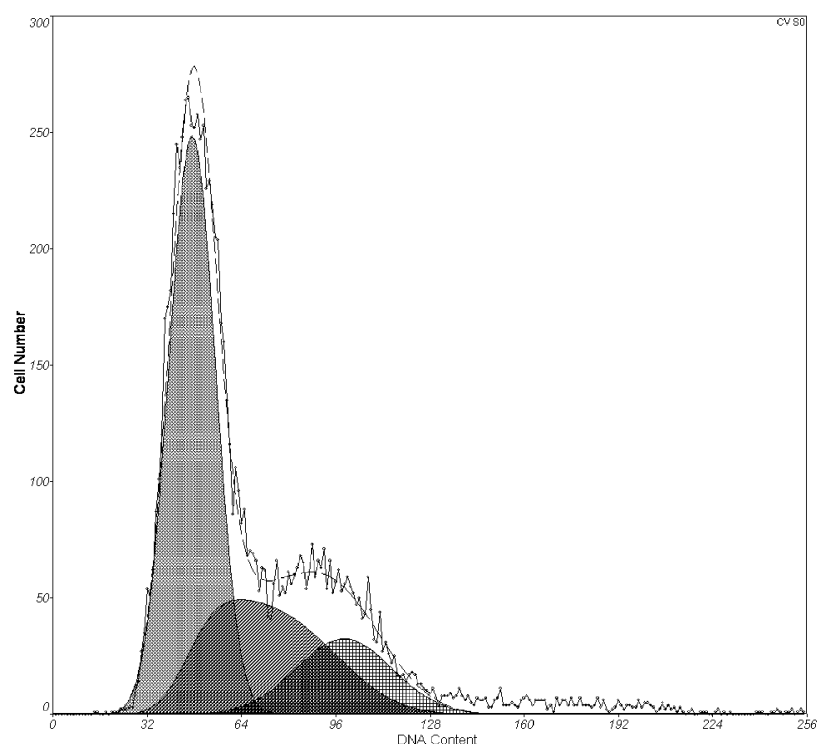

Fig. 10: Propidium iodide-based DNA analysis of DPSCs after reaching 40 population doublings. Percentage of cells in $\mathrm{S}-\mathrm{G}_{2}$ phase decreased to $44 \%( \pm 4 \%)$.
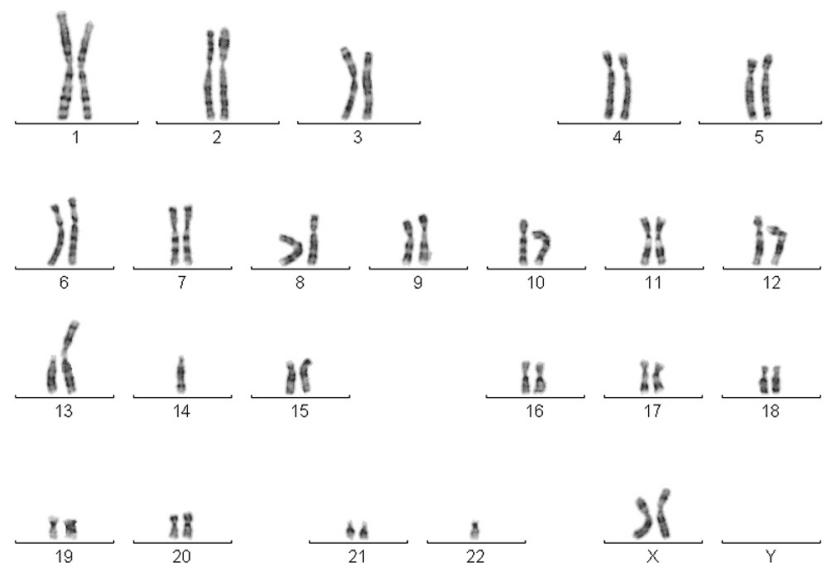

Fig. 11: In two experiments, lasting longer then 65 population doublings, 3 out of 100 evaluated mitoses were abnormal. Karyotype $44, \mathrm{XX}, \mathrm{t}(13,14),-22$ is shown. 
of DPSCs were inoculated on treated Cell ${ }^{+}$surface. Non-adherent cells and the remnants of pulp tissue (Fig. 5) were washed down using PBS 24 hours following inoculation. After 24 hours of cultivation, we observed first DPSCs, as a single cells or as a small colonies (Fig. 6). After 5 days, we found larger colonies in primary culture and cells were ready for first passaging (Fig. 7). Each following passaging was done after reaching $70 \%$ confluence.

We examined all basic biological characteristics (No. of population doublings, doubling time, plating efficiency, etc.) during long term cultivation of DPSCs. Compared with published data, we were the first authors, who expanded DPSCs over Hayflick's limit in a modified medium for MPCs.

Cumulated population doublings (PD) documented, that we have reached Hayflick's limit in all DPSCs cultures. Initial doubling time (DT) for first 40 population doublings (PD) was from 12 to 50 hours, after reaching 50 PD doubling time had increased to 60-90 hours (Graph 1). Plating efficiency of DPSCs from both compartments was $73.5 \pm 2.3 \%(68.1 \%-79.7 \%)$. Average viability of DPSCs was $96 \pm 3 \%(89 \%-100 \%)$. Diameter distribution of DPSCs showed stable lay-out - predominant population was $12-18 \mu \mathrm{m}$ in diameter (Graph 2). During long term cultivation we did not observe any signs of culture degeneration or spontaneous differentiation.

In addition, we observed some morphological differences between DPSCs from PVc and SOc (Figs. 8, 9). DPSCs from PVc were spindle-shaped cells with long processes in comparison with SOc DPSCs, which were more rounded. These morphological differences were not related to diameter distribution. DPSCs from both compartments showed similar number of uncumulated and cumulated population doublings.

Propidium iodide-based DNA analysis showed repeatedly $56 \%$ of DPSCs being in $\mathrm{S}_{2} \mathrm{G}_{2}$ phase of cell cycle. Percentage of cells in $\mathrm{S}_{-} \mathrm{G}_{2}$ phase decreased to $44 \% \pm 4 \%$ (Fig. 10) after reaching 40 population doublings.

DPSCs both primary cultures and cultures expanded over Hayflick's limit were cytogenetically stable. Cytogenetic examination of DPSCs showed normal karyotype in five consecutive experiments. As for karyotypes, we did not find any differences between PV and SO compartments. In two experiments, lasting longer then 65 population doublings, 3 out of 100 evaluated mitoses were abnormal (Fig. 11). We presume that abnormal mitoses are "artefacts" arising from prolonged in vitro cultivation.

In our study, we also isolated MPCs from bone marrow in order to compare DPSCs with MPCs. We used the same protocol for cultivation of MPCs and we obtained average $66 \times 10^{6} \pm 23 \times 10^{6}\left(11.6 \times 10^{6}-125 \times 10^{6}\right)$ of mononuclear cells from bone marrow. Cumulated population doublings documented, that we also reached Hayflick's limit with MPCs cultures. Initial doubling time for first 43 population doublings was from 12 to 50 hours, after reaching 55 PD, doubling time had increased to 60-90 hours. Plating effi-

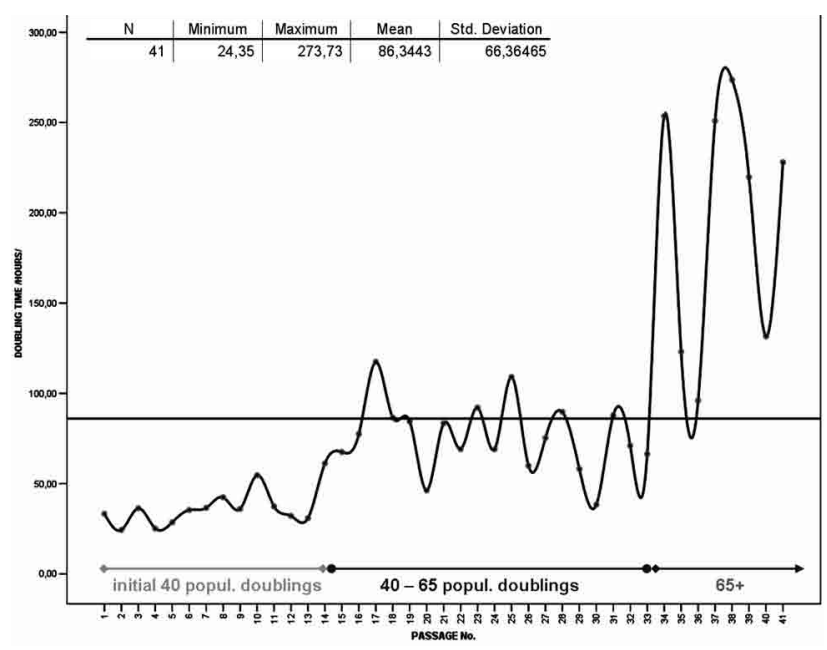

Graph 1: DPSCs doubling time trend. Initial doubling time (DT) for first 40 population doublings (PD) was from 12-50 hours, after reaching 50 PD doubling time had increased to 60-90 hours.

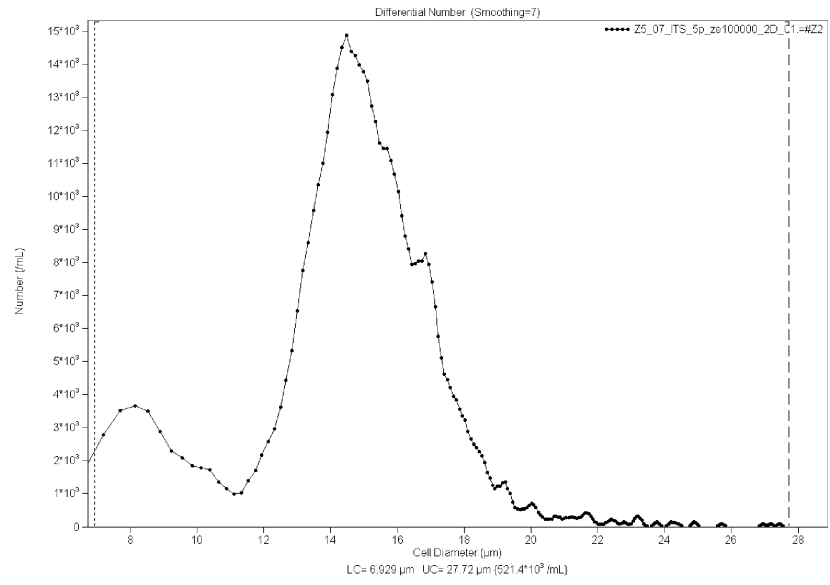

Graph 2: Diameter distrubution of DPSCs cultivated in MPCs medium. Predominant population is $12-18 \mu \mathrm{m}$ in diameter.

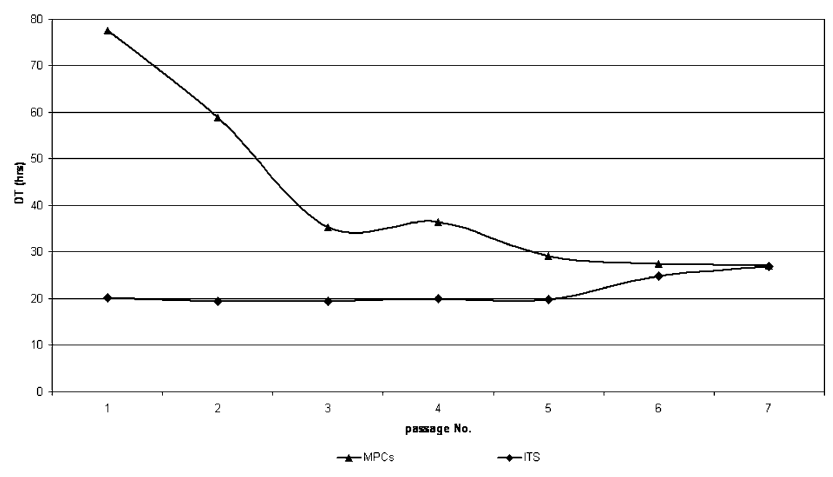

Graph 3: Doubling time analysis within firts 7 passages document advantage of using MPCs medium supplemented with ITS. Addition of ITS caused DT stabilization within initial passages and increased proliferation rate. 
ciency of MPCs was $69.3 \pm 2,7 \%(62.3-74.7 \%)$. Average viability of MPCs was $95 \pm 3 \%$ (85-99\%). Diameter distribution of MPCs showed stable curves - predominant population was $8-14 \mu \mathrm{m}$ in diameter. Signs of culture degeneration or spontaneous differentiation were not observed. DNA analysis showed constantly $25 \% \pm 6 \%$ of MPCs being in $\mathrm{S}-\mathrm{G}_{2}$ phase. All MPCs cultures were cytogenetically stable without abnormities in karyotype.

Moreover, we have analysed influence of ITS supplemented basal medium on DPSCs. Doubling time analysis within firts 7 passages (Graph 3) clearly showed advantage of using MPCs medium supplemented with ITS. Addition of ITS caused DT stabilization during initial passages and increased proliferation rate. DPSCs cultivated in MPCs medium supplemented with ITS reached Hayflick's limit at about $25 \pm 4$ days earlier than DPSCs cultivated in basic MPCs medium. DPSCs cultivated in ITS supplemented medium did not show any signs of degeneration or spontaneous differentiation. ITS supplement did not influence DPSCs morphology and average cell diameter.

\section{Discussion}

Dental pulp represents well delimited and from other tissues separated compartment, which retains unique histological structure and stem cell niche. Since there are two sources for dental pulp development (dental mesenchyme of neural crest origin and vascular mesenchyme) we suppose that in agreement with this there are two different lines of DPSCs inside the DP.

In our experiments we were able to isolate DPSCs from dental pulp of either permanent or exfoliated teeth using Luer's forceps or extirpation needle. On the contrary, we were not able to isolate DPSCs from the teeth which were splitted using skive or diamond grindstone. We suppose that DP was overheated and under severe mechanical stress in those cases.

Unlike other investigators $(2,7)$, we have cultivated undifferentiated DPSCs for long time, over 60 population doublings in cultivation media designed for bone marrow MPCs. After reaching Hayflick's limit, they still have normal karyotype, without any signs of genetic instability. We were the first investigators, who examined DPSCs doubling time. Initial doubling time of our cultures was from 12 to 50 hours for first 40 population doublings, after reaching 50 PD, doubling time had increased to 60-90 hours (Graph 1). Regression analysis of uncumulated population doublings proved tight dependence of population doublings on passage number and slow decrease of proliferation potential.

First published studies $(2,3,7)$ proposed that DPSCs were probably localized around DP blood vessels. In our experiments, we were able to isolate DPSCs from two DP compartments. We named these compartments according to their localization within the DP - subodontoblastic compartment (inner surface of tooth and outer part of DP) and perivascular compartment (the inner part of DP). DPSCs isolated from PVc were spindle-shaped with long processes. Conversely, DPSCs from SOc were more rounded. These morphological differences were not related to diameter distribution. DPSCs isolated from both discussed compartments showed similar number of uncumulated and cumulated population doublings.

In comparison with bone marrow MPCs, DPSCs share similar biological characteristics and stem cell properties. DNA analysis proved that DPSCs have more cells in $\mathrm{S}_{-} \mathrm{G}_{2}$ phase than bone marrow MPCs. Higher proliferation activity of DPSCs was confirmed by DT trend analysis. In addition, we did not observe any signs of spontaneous differentiation during DPSCs long term cultivation.

In addition, we have analysed influence of ITS supplemented basal medium on DPSCs. DPSCs cultivated in ITS supplemented medium reached Hayflick's limit at about 25 days $( \pm 4)$ earlier than DPSCs cultivated in basic MPCs medium and did not express any signs of degeneration or spontaneous differentiation. ITS supplement did not influence DPSCs morphology and cell diameter.

In our future experiments, we would like to focus on phenotypic analysis, differentiation potential and development of DPSCs isolated from both defined DP compartments.

\section{Conclusions}

We have isolated and ex vivo expanded 2 different populations of DPSCs from several adult teeth and one homogeneous population from exfoliated tooth beyond Hayflick's limit. Cultivated DPSCs and SHED were higly proliferative and cytogenetically stable stem cells. Morphological differences of cells isolated from both defined compartments were not related to changes in proliferation potential. Over the entire cultivation period, we did not observe any changes in cell viability and cells remained undifferentiated. Not only for mentioned reasons, dental pulp represents an alternative and easily accessible source for obtaining tissue-specific stem cells which are histocompatible with tissues of the individual patient.

\section{Acknowledgments}

The authors wish to thank V. Bartáková, M.D. for help with extraction of the tooth, H. Rückerová, for help in the tissue cultures laboratory and Mgr. Kučerová for karyotyping. Work was supported by grant project of the Ministry of Health, Czech Republic NR 9182-3/07, by the research project of the Ministry of Education, CR MSM 0021620820 and internal grant for $1^{\text {st }}$ year Ph.D. students, No. 84124 of Charles University in Prague, Faculty of Medicine in Hradec Králové.

\section{References}

\footnotetext{
1. Avery JK. Oral development and histology. $2^{\text {nd }}$ ed. New York: Thieme Medical Publishers, Inc., 1994:71-79.

2. Gronthos S, Cherman N, Robey P, Shi S. Human dental pulp stem cells. Adult Stem Cells. Totowa, New Jersey: Humana Press, 2004:37-51,101-49.
} 
3. Gronthos S, Mankani M, Brahim J et al. Postnatal human dental pulp stem cells (DPSCs) in vitro and in vivo. Proc Natl Acad Sci USA 2000;97:13625-30.

4. Hayflick L. The limited in vitro lifetime of human diploid cell strains. Exp. Cell Res. 1965: 2323-8.

5. Ivančaková R, Soukup T, Suchánek J, Karbanová J. Metodiky odběru zubn pulpy pro izolaci a kultivaci kmenových buněk. Čs. Stomat. 2006;106(5): $131-5$

6. Jiang Y, Jahagirdar BN, Reinhardt L, et al. Pluripotency of mesenchymal stem cells derived from adult marrow. Nature 2002;418(4):41-49 + Supplementary Information www.nature.com/nature

7. Miura M, Gronthos S, Zhao M, Lu B, Fisher LW, Robey PG, Shi S. SHED: Stem cells from human exfoliated deciduous teeth, Proc Natl Acad Sci USA 2003; 100:5807-12.
8. Minguel JJ, Erices A, Conget P. Mesenchymal stem cells. Exp Biol Med 2001; 226:507-20

9. Morsczeck C, Reichrt TE, Völlner F, Gerlach T, Driemel O. The state of the art in human dental stem cell research. Mund Kiefer Gesichtschir 2007 Sep 6.

10. Reyes M, Lund T, Lenvik T, Aguiar D, Koodie L, Verfaillie CM. Purification and ex vivo expansion of postnatal human marrow mesodermal progenitor cells. Blood 2001;98(9):2615-25.

11. Soukup T, Mokrý J, Karbanová J, Pytlík R, Suchomel P, Kučerová L. Mesenchymal stem cells isolated from the human bone marrow: cultivation, phenotypic analysis and changes in proliferation kinetics. Acta Medica (Hradec Kralove) 2006;49(1):27-33.

12. Turksen K. Adult Stem Cells. Totowa, New Jersey: Humana Press, 2004:37-51, $101-49$

Submitted March 2007.

Accepted August 2007.

\section{Corresponding author:}

Jakub Suchánek, M. D., Department of Dentistry, University Hospital,

Sokolská 581, 50005 Hradec Králové, Czech Republic, e-mail: suchanekj@lfhk.cuni.cz 